\title{
CHANGES OF VARIABLES NEAR A PERIODIC ORBIT
}

\author{
BY \\ AL KELLEY( $\left.{ }^{1}\right)$
}

1. Introduction. For a certain class of ordinary differential equations (see (1)) we construct changes of variables to obtain new systems of differential equations which exhibit the stable and unstable manifolds in a canonical way, and which linearize or normalize the equations when restricted to the stable or unstable manifold. Here we are concerned with systems which can be expanded in power series with variable coefficients. When the coefficients are constant our results in part reduce to those of Poincaré [6], Dulac [2], and C. L. Siegel [9]. Our results differ from those of Sibuya [7], [8], in that we have added a $\theta$-equation, included perturbation results, and have worked in the context of changes of variables.

In this paper $\theta$ is restricted to be a real scalar variable, but our main interest lies in the case when $\theta$ is a complex vector-valued variable. This case is discussed in [4]. The results depend heavily on the techniques developed here.

Although our results are closer to Sibuya's, our main theme, changes of variables, is based on the work of C. L. Siegel [9]. Our techniques are classical in nature and in certain cases some of our results overlap the recent (nonclassical in nature) work of Belaga [1] and C. L. Siegel [10]. A more adequate bibliography and history concerning changes of variables and linearization is given in [3].

This paper constitutes in part the author's thesis [3] written under the direction of Professor S. P. Diliberto.

2. Notation. Let $\theta$ be a real scalar, $z=\left(z_{1}, \ldots, z_{p}\right)$ a complex vector, and $q$ a nonnegative integer. We define $\Gamma^{q}(z)$ to be the class of functions which satisfy (i), (ii), and (iii) below.

(i) $F=F(\theta, z)$ is a complex vector-valued function or a complex matrix-valued function (dim $F$ unspecified) defined and continuous for all real $\theta$ and for $z$ in some neighborhood of $z=0$.

(ii) $F$ has a convergent power series expansion in $z$ (components of $z$ ) about the point $z=0$; the coefficients in the expansion are continuous, bounded, vectorvalued or matrix-valued (as the case may be) functions of $\theta$; there are no terms of degree less than $q$ in the expansion of $F$.

(iii) For some $K>0$ components of $F$ are majorized by

$$
K\left(z_{1}+\cdots+z_{p}\right)^{q}\left[1-K\left(z_{1}+\cdots+z_{p}\right)\right]^{-1}
$$

uniformly in $\theta$. The constant $K$ depends on $F$.

Received by the editors November 5, 1965 and, in revised form, July 7, 1966.

(1) This work was supported in part by the Office of Naval Research, the National Academy of Science, and National Science Foundation Grant GP2439. 
Given $G(\theta, z) \in \Gamma^{q}(z)$ there exists $G^{\prime}(z)$ (primes will always designate majorants) such that

$$
G(\theta, z) \ll G^{\prime}(z)
$$

where the symbol « means $G$ and $G^{\prime}$ belong to the same $\Gamma$ class, $\operatorname{dim} G=\operatorname{dim} G^{\prime}$, and $G$ is majorized componentwise by $G^{\prime}$ in the sense of Cauchy.

If $H \in \Gamma^{q}(z)$ is a vector or matrix whose coefficients in its expansion are continuously differentiable functions of $\theta$, and if the term-by-term derivative of $H$ with respect to $\theta$ also belongs to $\Gamma^{q}(z)$, then we write $H_{\theta} \in \Gamma^{q}(z)$.

3. Main theorems. Our interest lies in real systems of differential equations. However, in order to introduce the Jordan canonical form for the matrices involved ( $A$ and $B$ in (1) below), we lose the real character of the equations. Nevertheless, because we started with real equations, we may restrict $\theta$ to be real even though the differential equation for $\theta$ in (1) may be complex.

Consider the system of ordinary differential equations

$$
\begin{aligned}
\dot{\theta} & =1+\Theta(\theta, x, y) \\
\dot{x} & =A x+X(\theta, x, y) \\
\dot{y} & =B y+Y(\theta, x, y)
\end{aligned}
$$

where $A$ and $B$ are, respectively, $m \times m$ and $n \times n$ constant matrices in Jordan canonical form; $A=\operatorname{diag}\left(\lambda_{1}, \ldots, \lambda_{m}\right)+\operatorname{subdiag}\left(\gamma_{1}, \ldots, \gamma_{m-1}\right)$ with $\mathscr{R}\left(\lambda_{j}\right)<0$ and $\gamma_{j}=0$ or $\gamma(j=1, \ldots, m) ; B=\operatorname{diag}\left(\mu_{1}, \ldots, \mu_{n}\right)+\operatorname{subdiag}\left(\delta_{1}, \ldots, \delta_{n-1}\right)$ with $\mathscr{R}\left(\mu_{j}\right) \geqq 0$ and $\delta_{j}=0$ or $\delta(j=1, \ldots, n) ; \gamma$ and $\delta$ are sufficiently small positive constants (to be specified below); $\theta$ is a real scalar; $x$ and $y$ are complex vectors; $\Theta \in \Gamma^{1}(x, y) ; X, Y \in \Gamma^{2}(x, y)$. In addition we assume with no loss of generality that the eigenvalues of $A$ have the following ordering

$$
\mathscr{R}\left(\lambda_{m}\right) \leqq \mathscr{R}\left(\lambda_{m-1}\right) \leqq \cdots \leqq \mathscr{R}\left(\lambda_{1}\right)<0 .
$$

Let $\alpha=\left(\alpha_{1}, \ldots, \alpha_{m}\right)$ represent $m$-tuples of nonnegative integers and define $|\alpha|=\alpha_{1}+\cdots+\alpha_{m}$. Let $\left(C_{j}\right), 1 \leqq j \leqq m$, represent the following condition on $\alpha$.

$$
\left(C_{j}\right): \mathscr{R}\left[\sum_{l=1}^{m} \alpha_{l} \lambda_{l}-\lambda_{j}\right] \neq 0 .
$$

Notice that (2) implies $\left(C_{1}\right)$ holds for all $|\alpha|>1$.

THEOREM 1. If conditions $\left(C_{j}\right)(j=2, \ldots, m)$ hold for all $|\alpha|>1$, then for system (1) there exists a unique change of variables

$$
\begin{aligned}
& u=x-P(\theta, x) \\
& v=y-Q(\theta, x)
\end{aligned}
$$


such that

$$
\begin{aligned}
\dot{u} & =A u+U(\theta, u, v) \\
\dot{v} & =B v+V(\theta, u, v) \\
U(\theta, u, 0) & \equiv 0, \quad V(\theta, u, 0) \equiv 0
\end{aligned}
$$

where $P, P_{\theta}, Q, Q_{\theta} \in \Gamma^{2}(x) ; U, V \in \Gamma^{2}(u, v)$. Moreover, if system (1) is periodic (almost periodic) in $\theta$, then the change of variables (3) and the transformed system (4) are also periodic (almost periodic) in $\theta$ with the same period (frequencies).

Before giving the proof of Theorem 1, let us describe what alterations occur when not all conditions $\left(C_{j}\right)(j=2, \ldots, m)$ hold for all $|\alpha|>1$.

THEOREM 2. If some condition $\left(C_{j}\right), 2 \leqq j \leqq m$, does not hold for all $|\alpha|>1$, then for system (1) there exists a nonunique change of variables

$$
\begin{aligned}
& u=x-P(\theta, x) \\
& v=y-Q(\theta, x)
\end{aligned}
$$

such that

$$
\begin{aligned}
\dot{u} & =A u+U(\theta, u, v) \\
\dot{v} & =B v+V(\theta, u, v) \\
U(\theta, u, 0) & =\tilde{U}(\theta, u), \quad V(\theta, u, 0) \equiv 0
\end{aligned}
$$

where the components of $\tilde{U}=\left(\tilde{U}_{1}, \ldots, \tilde{U}_{m}\right)$ are polynomials of the form

$$
\tilde{U}_{1} \equiv 0 \text {, }
$$

$$
\tilde{U}_{j}(\theta, u)=\sum \tilde{U}_{j, \alpha_{1}, \ldots, \alpha_{j-1}}(\theta) u_{1}^{\alpha} \cdots u_{j-1}^{\alpha_{j}-1} \quad(j=2, \ldots, m)
$$

the summation in (5) being taken over all $|\alpha|>1$ which do not satisfy condition $\left(C_{f}\right)$. The properties of $P, Q$, and $V$, and the remaining properties of $U$ are the same as those in Theorem 1.

Proof of Theorem 1. Suppose $P, Q, U$, and $V$ exist as formal power series with undetermined coefficients. Differentiating both sides of (3), we find that (1), (3), and (4) imply

$$
\begin{aligned}
& \dot{P}=A P+X-U, \\
& \dot{Q}=B Q+Y-V .
\end{aligned}
$$

From (3) we see that $v=0$ if and only if $y=Q(\theta, x)$. Putting $v=0$ in (6) and using the property $U(\theta, u, 0) \equiv 0, V(\theta, u, 0) \equiv 0$ given in (4), we find that (6) reduces to

$$
\begin{aligned}
& P_{\theta}\{1+\Theta(\theta, x, Q)\}+P_{x}\{A x+X(\theta, x, Q)\}=A P+X(\theta, x, Q) \\
& Q_{\theta}\{1+\Theta(\theta, x, Q)\}+Q_{x}\{A x+X(\theta, x, Q)\}=B Q+Y(\theta, x, Q)
\end{aligned}
$$


or, equivalently,

$$
\begin{aligned}
\frac{\partial}{\partial \theta} P_{j}+\sum_{l=1}^{m} \lambda_{l} x_{l} & \frac{\partial}{\partial x_{l}} P_{j}-\lambda_{j} P_{j} \\
= & \delta_{j-1} P_{j-1}-\sum_{l=1}^{m} \delta_{l-1} x_{l-1} \frac{\partial}{\partial x_{l}} P_{j}-\Theta(\theta, x, Q) \frac{\partial}{\partial \theta} P_{l} \\
& +X_{j}(\theta, x, Q)-\sum_{l=1}^{m} X_{l}(\theta, x, Q) \frac{\partial}{\partial x_{l}} P_{j} \quad(j=1, \ldots, m),
\end{aligned}
$$$$
\frac{\partial}{\partial \theta} Q_{j}+\sum_{l=1}^{m} \lambda_{l} x_{l} \frac{\partial}{\partial x_{l}} Q_{j}-\mu_{j} Q_{j}
$$

$$
\begin{aligned}
& =\gamma_{j-1} Q_{j-1}-\sum_{l=1}^{m} \delta_{l-1} x_{l-1} \frac{\partial}{\partial x_{l}} Q_{j}-\Theta(\theta, x, Q) \frac{\partial}{\partial \theta} Q_{j} \\
& \quad+Y_{j}(\theta, x, Q)-\sum_{l=1}^{m} X_{l}(\theta, x, Q) \frac{\partial}{\partial x_{l}} Q_{j} \quad(j=1, \ldots, n),
\end{aligned}
$$

where $\delta_{0}=\gamma_{0}=0$. Notice that (7) and (9) contain both $P$ and $Q$, but (8) and (10) contain only $Q$. For this reason we solve (8), or equivalently (10), first. Consider $Q$ as a formal power series with undetermined coefficients.

$$
Q_{j}(\theta, x)=\sum_{|\alpha|>1} Q_{j, \alpha}(\theta) x^{\alpha} \quad(j=1, \ldots, n)
$$

where $x^{\alpha}=x_{1}^{\alpha} \cdots x_{m}^{\alpha_{m}}$. With this notation we have from (10)

$$
\frac{\partial}{\partial \theta} Q_{j, \alpha}+\mu_{j, \alpha} Q_{j, \alpha}=Z_{j, \alpha}
$$

where

$$
\mu_{j, \alpha}=\sum_{l=1}^{m} \alpha_{l} \lambda_{l}-\mu_{j}
$$

and $Z_{j, \alpha}=Z_{j, \alpha}(\theta)$ represents the coefficient of $x^{\alpha}$ in the right side of (10). Since by hypothesis $\mathscr{R}\left(\lambda_{l}\right)<0$ and $\mathscr{R}\left(\mu_{j}\right) \geqq 0$, it follows that $\mathscr{R}\left(\mu_{j, \alpha}\right)<0$; and hence, by Lemma 1 (below) if $Z_{j, \alpha}$ is a bounded, continuous function of $\theta$, then (11) determines $Q_{j, \alpha}$ uniquely as a bounded, continuously differentiable function of $\theta$. Moreover, if $Z_{j, \alpha}$ is periodic (almost periodic) in $\theta$, then $Q_{j, \alpha}$ also is periodic (almost periodic) with the same period (frequencies). Thus, by following along the order relation $\prec$ (below), the coefficients of $Q$ are computed recursively from (10).

Order relation. Let $\prec$ represent the following order relation on subscripts. If $|\alpha|<\left|\alpha^{\prime}\right|$, then $(j, \alpha) \prec\left(j^{\prime}, \alpha^{\prime}\right)$. If $|\alpha|=\left|\alpha^{\prime}\right|$ and $j<j^{\prime}$, then $(j, \alpha) \prec\left(j^{\prime}, \alpha^{\prime}\right)$. If $|\alpha|=\left|\alpha^{\prime}\right|$ and $j=j^{\prime}$, then $\left(j, \alpha_{1}, \ldots, \alpha_{m}\right) \prec\left(j, \alpha_{1}, \ldots, \alpha_{l-1}+1, \alpha_{l}-1, \ldots, \alpha_{m}\right)$. 
Lemma 1. Consider the one-dimensional differential equation

$$
\dot{z}=a z+f(t),
$$

where $a$ is a complex number, $\mathscr{R}(a) \neq 0 ; f(t)$ is a bounded, continuous, complex valued function of $t$ defined for all $t$ real. The unique bounded solution of (12) is given by $z=\phi(t)$,

$$
\phi(t)=\int_{ \pm \infty}^{0} e^{-a \sigma} f(t+\sigma) d \sigma
$$

where the lower limit on the integral is chosen $+\infty$ if $\mathscr{R}(a)>0$ and $-\infty$ if $\mathscr{R}(a)<0$. Moreover, if $f(t)$ is periodic (almost periodic), then $\phi(t)$ also is periodic (almost periodic) with the same period (frequencies). From (12) and (13) we have the following estimates on the size of $\phi$ and $\dot{\phi}$.

$$
\begin{aligned}
& \|\phi\| \leqq|\mathscr{R}(a)|^{-1}\|f\|, \\
& \|\dot{\phi}\| \leqq\left\{|a||\mathscr{R}(a)|^{-1}+1\right\}\|f\|,
\end{aligned}
$$

where $\|\cdot\|=\sup _{t}|\cdot|$.

The proof of Lemma 1 is straightforward.

Having established $Q$ as a formal power series with coefficients which are continuously differentiable vector-valued functions of $\theta$, we must show that the power series $Q$ and its term-by-term derivative $Q_{\theta}$ converge. But notice from (8) that the convergence of $Q$ implies the convergence of $Q_{\theta}$. To show the convergence of $Q$ we will need

LEMma 2. For some $K>0$ sufficiently large the inequalities

$$
\begin{aligned}
\left|\mu_{j, \alpha}\right|\left|\mathscr{R}\left(\mu_{j, \alpha}\right)\right|^{-1}+1 & <K \\
\left|\mathscr{R}\left(\mu_{j, \alpha}\right)\right|^{-1} & <K|\alpha|^{-1}
\end{aligned}
$$

hold for $(j=1, \ldots, n)$ and all $|\alpha|>1$.

The proof of (15) is elementary, and the proof of (16) is classical. One can easily adapt the arguments involved in the inequality (2.1) on page 109 of [5] to show our inequality (16).

Choose $\Theta^{\prime}, X^{\prime}$, and $Y^{\prime}$ such that $\Theta(\theta, x, y) \ll \Theta^{\prime}(x, y), X(\theta, x, y) \ll X^{\prime}(x, y)$, and $Y(\theta, x, y) \ll Y^{\prime}(x, y)$. Let $Q^{\prime}=Q^{\prime}(x), \operatorname{dim} Q^{\prime}=\operatorname{dim} Q$, be the formal power series defined by

$$
\begin{array}{r}
\sum_{l=1}^{m} x_{l} \frac{\partial}{\partial x_{l}} Q_{j}^{\prime}=K\left[\gamma_{j-1} Q_{j-1}^{\prime}+\sum_{l=1}^{m} \delta_{l-1} x_{l-1} \frac{\partial}{\partial x_{l}} Q_{j}^{\prime}+\Theta^{\prime}\left(x, Q^{\prime}\right) \sum_{l=1}^{m} x_{l} \frac{\partial}{\partial x_{l}} Q_{j}^{\prime}\right. \\
\left.+Y_{j}^{\prime}\left(x, Q^{\prime}\right)+\sum_{l=1}^{m} X_{l}^{\prime}\left(x, Q^{\prime}\right) \frac{\partial}{\partial x_{l}} Q_{j}^{\prime}\right] \quad(j=1, \ldots, n) .
\end{array}
$$


The coefficients of $Q^{\prime}$ are computed recursively from (17) in a manner similar to the way that the coefficients of $Q$ were computed from (10). Clearly the coefficients of $Q_{j}^{\prime}(j=1, \ldots, n)$ are nonnegative constants. Let

$$
Q_{j}^{\prime}(x)=\sum_{|\alpha|>1} Q_{j, \alpha}^{\prime} x^{\alpha} \quad(j=1, \ldots, n)
$$

represent the formal power series for $Q^{\prime}$. From (17)

$$
Q_{j, \alpha}^{\prime}=K|\alpha|^{-1} Z_{j, \alpha}^{\prime}
$$

where $K Z_{j, \alpha}^{\prime}$ represents the coefficient of $x^{\alpha}$ in the right side of (17). Let $\|\cdot\|=\sup _{\theta}|\cdot|$ and suppose

$$
\left\|Z_{j, \alpha}\right\| \leqq Z_{j, \alpha}^{\prime}
$$

From (11) and Lemma 1

and

$$
\left\|Q_{j, \alpha}\right\| \leqq\left|\mathscr{R}\left(\mu_{j, \alpha}\right)\right|^{-1}\left\|Z_{j, \alpha}\right\|
$$

$$
\left\|\frac{\partial}{\partial \theta} Q_{j, \alpha}\right\| \leqq\left\{\left|\mu_{j, \alpha}\right| \cdot\left|\mathscr{R}\left(\mu_{j, \alpha}\right)\right|^{-1}+1\right\}\left\|Z_{j, \alpha}\right\| .
$$

Thus from Lemma 2, (18), and (19)

and

$$
\left\|Q_{j, \alpha}\right\| \leqq Q_{j, \alpha}^{\prime}
$$

$$
\left\|\frac{\partial}{\partial \theta} Q_{j, \alpha}\right\| \leqq|\alpha| Q_{j, \alpha}^{\prime}
$$

Since (19) holds for $|\alpha|=2$, it follows by induction that

$$
Q(\theta, x) \ll Q^{\prime}(x) .
$$

Let $\zeta$ be a one-dimensional variable. Clearly, when we put $\zeta=x_{1}+\cdots+x_{m}$,

$$
Q^{\prime}\left(x_{1}, \ldots, x_{m}\right) \ll Q^{\prime}(\zeta, \ldots, \zeta) .
$$

Define

$$
\Theta^{\prime \prime}(\zeta, y)=\Theta^{\prime}(\zeta, \ldots, \zeta, y), X^{\prime \prime}(\zeta, y)=X^{\prime}(\zeta, \ldots, \zeta, y)
$$

and

$$
Y^{\prime \prime}(\zeta, y)=Y^{\prime}(\zeta, \ldots, \zeta, y)
$$

where we have replaced $x_{l}(l=1, \ldots, m)$ by $\zeta$ in $\Theta^{\prime}, X^{\prime}$, and $Y^{\prime}$. If in (17) we put $x_{1}=x_{2}=\cdots=x_{m}=\zeta$, then we obtain

$$
\begin{aligned}
\zeta \sum_{l=1}^{m} \frac{\partial}{\partial x_{l}} Q_{j}^{\prime}=K\left[\gamma_{j-1} Q_{j-1}^{\prime}+\zeta \sum_{l=1}^{m} \delta_{l-1} \frac{\partial}{\partial x_{l}} Q_{j}^{\prime}+\Theta^{\prime \prime}\left(\zeta, Q^{\prime}\right) \zeta \sum_{l=1}^{m} \frac{\partial}{\partial x_{l}} Q_{j}^{\prime}\right. \\
\left.+Y^{\prime \prime}\left(\zeta, Q^{\prime}\right)+\sum_{l=1}^{m} X_{l}^{\prime \prime}\left(\zeta, Q^{\prime}\right) \frac{\partial}{\partial x_{l}} Q_{j}^{\prime}\right] \quad(j=1, \ldots, n)
\end{aligned}
$$


where $Q^{\prime}=Q^{\prime}(\zeta, \ldots, \zeta)$. Define $Q^{\prime \prime}=Q^{\prime \prime}(\zeta), \operatorname{dim} Q^{\prime \prime}=\operatorname{dim} Q^{\prime}$, by the functional equation

$$
\begin{aligned}
\zeta \frac{d}{d \zeta} Q_{j}^{\prime \prime}-Q_{j}^{\prime \prime}=2 K\left[\Theta^{\prime \prime}\left(\zeta, Q^{\prime \prime}\right) \zeta \frac{d}{d \zeta} Q_{j}^{\prime \prime}+Y_{j}^{\prime \prime}\left(\zeta, Q^{\prime \prime}\right)+X_{1}^{\prime \prime}\left(\zeta, Q^{\prime \prime}\right) \frac{d}{d \zeta} Q_{j}^{\prime \prime}\right] \\
(j=1, \ldots, n) .
\end{aligned}
$$

For the purpose of showing that $Q^{\prime \prime}(\zeta)$ majorizes $Q^{\prime}(\zeta, \ldots, \zeta)$ we write (21) as

$$
\zeta \frac{d}{d \zeta} Q_{j}^{\prime \prime}=\frac{1}{2} Q_{j}^{\prime \prime}+\frac{1}{2} \zeta \frac{d}{d \zeta} Q_{j}^{\prime \prime}+K[\cdots]
$$

where $[\cdots]$ represents the expression in brackets in the right side of (21). With no loss of generality we may assume that

$$
\max [K \gamma, K \delta]<\frac{1}{2} .
$$

Furthermore, with no loss of generality we may assume that $X_{1}^{\prime}=\cdots=X_{m}^{\prime}$ and $Y_{1}^{\prime}=\cdots=Y_{n}^{\prime}$. Thus $X_{1}^{\prime \prime}=\cdots=X_{m}^{\prime \prime}$ and $Y_{1}^{\prime \prime}=\cdots=Y_{n}^{n}$, and it follows that $Q_{1}^{\prime \prime}=\cdots=Q_{n}^{\prime \prime}$. A direct comparison of (20) and (22) along with the additional fact that

$$
\sum_{l=1}^{m} \frac{\partial}{\partial x_{l}} Q_{j}^{\prime}(\zeta, \ldots, \zeta)=\frac{d}{d \zeta} Q_{j}^{\prime}
$$

now shows that

$$
Q^{\prime}(\zeta, \ldots, \zeta) \ll Q^{\prime \prime}(\zeta)
$$

At this point it is convenient to write (21) in vector notation.

$$
\zeta Q_{\zeta}^{\prime \prime}-Q^{\prime \prime}=2 K\left[\Theta^{\prime \prime}(\zeta, Q) \zeta Q_{\zeta}^{\prime \prime}+Y^{\prime \prime}\left(\zeta, Q^{\prime \prime}\right)+X_{1}^{\prime \prime}\left(\zeta, Q^{\prime \prime}\right) Q_{\zeta}^{\prime \prime}\right]
$$

Let $Q^{n}=\zeta Q^{*}$. From (23) we obtain

$$
Q_{\zeta}^{*}=2 K \zeta^{-2}\left[\Theta^{\prime \prime}\left(\zeta, \zeta Q^{*}\right)\left\{\zeta Q^{*}+\zeta^{2} Q_{\zeta}^{*}\right\}+Y^{\prime \prime}\left(\zeta, \zeta Q^{*}\right)+X_{1}^{\prime \prime}\left(\zeta, \zeta Q^{*}\right)\left\{Q^{*}+\zeta Q_{\zeta}^{*}\right\}\right] .
$$

Since $\Theta^{\prime \prime}(\zeta, y) \in \Gamma^{1}(\zeta, y)$ and $X^{\prime \prime}(\zeta, y), Y^{\prime \prime}(\zeta, y) \in \Gamma^{2}(\zeta, y)$, one easily puts (24) in the form

$$
Q_{\zeta}^{*}=G\left(\zeta, Q^{*}\right)
$$

where $G$ is analytic in $\left(\zeta, Q^{*}\right)$ in some neighborhood of $\zeta=0, Q^{*}=0$. Since analytic systems of differential equations have analytic solutions, (25) or (24) determines $Q^{*}=Q^{*}(\zeta), Q^{*}(0)=0$ as an analytic function of $\zeta$ in some neighborhood of $\zeta=0$. For $\zeta=x_{1}+\cdots+x_{m}$ we have shown that $Q(\theta, x) \ll Q^{\prime}(\zeta, \ldots, \zeta) \ll Q^{\prime \prime}(\zeta)=\zeta Q^{*}(\zeta)$. Hence, $Q(\theta, x) \in \Gamma^{2}(x)$ and, by our earlier remark, $\partial Q(\theta, x) / \partial \theta \in \Gamma^{2}(x)$.

The construction and convergence of $P$ follows a similar pattern. Let

$$
P_{f}(\theta, x)=\sum_{|\alpha|>1} P_{f, \alpha}(\theta) x^{\alpha} \quad(j=1, \ldots, m)
$$


represent the expansion of $P$ with undetermined coefficients. From (9)

$$
\frac{\partial}{\partial \theta} P_{j, \alpha}+\lambda_{j, \alpha} P_{j, \alpha}=Z_{j, \alpha}
$$

where

$$
\lambda_{j, \alpha}=\sum_{l=1}^{m} \alpha_{l} \lambda_{l}-\lambda_{j}
$$

and $Z_{j, \alpha}$ represents the coefficient of $x^{\alpha}$ in the right side of (9). In order to compute $P_{j, \alpha}$ by means of Lemma 1 , we require that $\mathscr{R}\left(\lambda_{j, \alpha}\right) \neq 0$. This inequality is assured by the hypothesis that conditions $\left(C_{j}\right)(j=2, \ldots, m)$ hold for all $|\alpha|>1$. Therefore, by following along the order relation $\prec$, we can compute the coefficients of $P$ recursively. Moreover, as before, these coefficients will reflect the periodic (almost periodic) properties of system (1). The proof of the convergence of $P$ is similar to the proof of the convergence of $Q$, and the convergence of $P_{\theta}$ then is implied by (7). The existence of $U$ and $V$ with the stated properties is implied by the existence of $P$ and $Q$. This completes the proof of Theorem 1 .

Proof of Theorem 2. Because of the ordering (2) of the eigenvalues of $A$, if $\alpha=\left(\alpha_{1}, \ldots, \alpha_{m}\right),|\alpha|>1$, does not satisfy condition $\left(C_{j}\right)$, then $\alpha_{j}=\alpha_{j+1}=\cdots=\alpha_{m}=0$. Thus $\tilde{U}$ has the form given in (5). The equation that $P$ must satisfy now will be the same as (9) with the term $-\tilde{U}_{j}(\theta, x-P)$ added to the right side. As before, the coefficients of $P$ are computed recursively from (26), except that whenever $\mathscr{R}\left(\lambda_{j, \alpha}\right)=0$ we choose the coefficient $\tilde{U}_{j, \alpha}$ such that $Z_{j, \alpha}$ vanishes. This allows us to take $P_{j, \alpha} \equiv 0$ as a solution. At this point nonuniqueness occurs because other choices for $\tilde{U}_{j, \alpha}$ and $P_{j, \alpha}$ also suffice. Since $\tilde{U}$ is a polynomial, it has a majorant, and therefore in constructing a majorant for $P$ there are no new difficulties. This completes the proof of Theorem 2.

The equation $y=Q(\theta, x)$ describes the stable manifold of system (1), whereas the change of variables $u=x-P(\theta, x)$ accomplishes a linearization or normalization of system (1) when restricted to the stable manifold. In an entirely analogous manner we can find the unstable manifold of the new system

$$
\begin{aligned}
& \dot{\theta}=1+\Theta(\theta, u, v) \\
& \dot{u}=A u+U(\theta, u, v) \\
& \dot{v}=B v+V(\theta, u, v)
\end{aligned}
$$

and construct a change of variables which linearizes or normalizes this system when restricted to the unstable manifold. The composition of two changes of variables, the first given in Theorem 1 or 2 and the second analogous but constructed with respect to the unstable manifold of system (27), gives the proof of Theorem 3 (below) in the general case. 
Consider the system

$$
\begin{aligned}
\dot{\theta} & =1+\Theta(\theta, x, y, z) \\
\dot{x} & =A x+X(\theta, x, y, z) \\
\dot{y} & =B y+Y(\theta, x, y, z) \\
\dot{z} & =C z+Z(\theta, x, y, z)
\end{aligned}
$$

where $A, B$, and $C$ are constant matrices in Jordan canonical form;

$$
A=\operatorname{diag}\left(\lambda_{1}, \ldots, \lambda_{k}\right)+\operatorname{subdiag}\left(\gamma_{1}, \ldots, \gamma_{k-1}\right)
$$

with $\mathscr{R}\left(\lambda_{j}\right)<0$ and $\gamma_{j}=0$ or $\gamma(j=1, \ldots, k)$;

$$
B=\operatorname{diag}\left(\mu_{1}, \ldots, \mu_{m}\right)+\operatorname{subdiag}\left(\delta_{1}, \ldots, \delta_{m-1}\right)
$$

with $\mathscr{R}\left(\mu_{j}\right)=0$ and $\delta_{j}=0$ or $\delta(j=1, \ldots, m)$;

$$
C=\operatorname{diag}\left(\nu_{1}, \ldots, v_{n}\right)+\operatorname{subdiag}\left(\varepsilon_{1}, \ldots, \varepsilon_{n-1}\right)
$$

with $\mathscr{R}\left(\nu_{j}\right)>0$ and $\varepsilon_{j}=0$ or $\varepsilon(j=1, \ldots, n) ; \gamma, \delta$, and $\varepsilon$ are sufficiently small positive constants; $\theta$ is a real scalar; $x, y$, and $z$ are complex vectors; $\Theta \in \Gamma^{1}(x, y, z)$; $X, Y, Z \in \Gamma^{2}(x, y, z)$. In addition we assume with no loss of generality that the eigenvalues of $A$ and $C$ have the following order.

$$
\mathscr{R}\left(\lambda_{k}\right) \leqq \cdots \leqq \mathscr{R}\left(\lambda_{1}\right)<0<\mathscr{R}\left(\nu_{1}\right) \leqq \cdots \leqq \mathscr{R}\left(v_{n}\right)
$$

THEOREM 3. For system (28) there exists a change of variables

$$
\begin{aligned}
u & =x-P(\theta, x, z) \\
v & =y-Q(\theta, x, z) \\
w & =z-R(\theta, x, z)
\end{aligned}
$$

such that

$$
\begin{aligned}
\dot{u} & =A u+U(\theta, u, v, w) \\
\dot{v} & =B v+V(\theta, u, v, w) \\
\dot{w} & =C w+W(\theta, u, v, w) \\
U(\theta, 0,0, w) & \equiv 0, U(\theta, u, 0,0)=\tilde{U}(\theta, u) \\
V(\theta, 0,0, w) & \equiv V(\theta, u, 0,0) \equiv 0 \\
W(\theta, 0,0, w) & =\tilde{W}(\theta, w), W(\theta, u, 0,0) \equiv 0
\end{aligned}
$$

where $P, P_{\theta}, Q, Q_{\theta}, R, R_{\theta} \in \Gamma^{2}(x, z) ; U, V, W \in \Gamma^{2}(u, v, w)$; the components of $\tilde{U}$ are polynomials of the form

$$
\begin{aligned}
\tilde{U}_{1} & \equiv 0 \\
\tilde{U}_{j}(\theta, u) & =\sum \tilde{U}_{j, \alpha_{1}, \ldots, \alpha_{j-1}}(\theta) u_{1}^{\alpha} \cdots u_{j-1}^{\alpha_{j}} \quad(j=2, \ldots, k)
\end{aligned}
$$


the summation being taken over all $k$-tuples of nonnegative integers $\alpha=\left(\alpha_{1}, \ldots, \alpha_{k}\right)$, $|\alpha|>1$, which do not satisfy condition $\left(C_{j}^{1}\right)$ below; the components of $\tilde{W}$ are polynomials of the form

$$
\begin{aligned}
\tilde{W}_{1} & \equiv 0 \\
\tilde{W}_{j}(\theta, w) & =\sum \tilde{W}_{j, \beta_{1}, \ldots, \beta_{j-1}}(\theta) w_{1}^{\beta_{1}} \ldots w_{j-1}^{\beta_{j}-1} \quad(j=2, \ldots, n)
\end{aligned}
$$

the summation being taken over all $n$-tuples of nonnegative integers $\beta=\left(\beta_{1}, \ldots, \beta_{n}\right)$, $|\beta|>1$, which do not satisfy condition $\left(C_{j}^{2}\right)$ below. If conditions $\left(C_{j}^{1}\right)(j=2, \ldots, k)$ hold for all $|\alpha|>1$, then $\widetilde{U} \equiv 0$. If conditions $\left(C_{j}^{2}\right)(j=2, \ldots, n)$ hold for all $|\beta|>1$, then $\tilde{W} \equiv 0$.

$$
\begin{aligned}
& \left(C_{j}^{1}\right): \mathscr{R}\left[\sum_{l=1}^{k} \alpha_{l} \lambda_{l}-\lambda_{j}\right] \neq 0, \\
& \left(C_{j}^{2}\right): \mathscr{R}\left[\sum_{l=1}^{n} \beta_{l} \nu_{l}-\nu_{j}\right] \neq 0 .
\end{aligned}
$$

When the coefficients of $\Theta, X, Y$, and $Z$ in system (28) are periodic (almost periodic) in $\theta$, then the coefficients of the change of variables and of the transformed system are also periodic (almost periodic) in $\theta$ with the same period (frequencies). If the coefficients in (28) have period $\omega$ in $\theta$, conditions $\left(C_{j}^{1}\right)$ and $\left(C_{j}^{2}\right)$ can be replaced by conditions $\left(C_{j}^{1}\right.$ period $\left.\omega\right)$ and $\left(C_{j}^{2}\right.$ period $\left.\omega\right)$, respectively.

$$
\begin{aligned}
& \left(C_{j}^{1} \text { period } \omega\right): \sum_{l=1}^{k} \alpha_{l} \lambda_{l}-\lambda_{j} \neq 2 \pi i \omega^{-1} \times \text { integer, } \\
& \left(C_{j}^{2} \text { period } \omega\right): \sum_{l=1}^{n} \beta_{l} \nu_{l}-\nu_{j} \neq 2 \pi i \omega^{-1} \times \text { integer } .
\end{aligned}
$$

When the coefficients in (28) are constant, the coefficients of the change of variables and of the transformed system also are constant; and conditions $\left(C_{j}^{1}\right)$ and $\left(C_{j}^{2}\right)$ can be replaced by conditions $\left(C_{j}^{1}\right.$ constant $)$ and $\left(C_{j}^{2}\right.$ constant $)$, respectively.

$$
\begin{aligned}
& \left(C_{j}^{1} \text { constant }\right): \sum_{l=1}^{k} \alpha_{l} \lambda_{l}-\lambda_{j} \neq 0, \\
& \left(C_{j}^{2} \text { constant }\right): \sum_{l=1}^{n} \beta_{l} \nu_{l}-\nu_{j} \neq 0 .
\end{aligned}
$$

Proof. As mentioned above, the composition of two changes of variables based on Theorems 1 and 2 accomplishes the proof of Theorem 3 in the general case. The fact that conditions $\left(C_{j}^{1}\right)$ and $\left(C_{j}^{2}\right)$ can be replaced by conditions $\left(C_{j}^{1}\right.$ period $\left.\omega\right)$ and $\left(C_{j}^{2}\right.$ period $\left.\omega\right)$, respectively, when the coefficients in (28) have period $\omega$ in $\theta$ follows from Lemma 3 below. When the coefficients in (28) are constant, the conditions ( $C_{j}^{1}$ constant) and $\left(C_{j}^{2}\right.$ constant) determine whether or not coefficients in the changes of variables are computable. Thus these conditions can replace $\left(C_{j}^{1}\right)$ 
and $\left(C_{j}^{2}\right)$, respectively. Convergence proofs are only slightly modified when these conditions for the periodic and constant case are used. This completes the proof of Theorem 3.

LEMMA 3. Consider the one-dimensional differential equation

$$
\dot{z}=a z+f(t)
$$

where $a$ is a constant and $f(t)$ is a complex-valued continuous function with period $\omega$ in $t$. If $a \neq 2 \pi i \omega^{-1} \times$ integer, then the unique solution $z=\phi(t)$ of (29) with period $\omega$ in $t$ is given by

$$
\phi(t)=\left(e^{-a \omega}-1\right)^{-1} \int_{0}^{\omega} e^{-a \sigma} f(t+\sigma) d \sigma
$$

The proof of this lemma is not difficult and is left to the reader.

4. Perturbation theory. Consider the system

$$
\begin{aligned}
\dot{\theta} & =1+\Theta(\theta, x, y, \varepsilon) \\
\dot{x} & =A x+X(\theta, x, y, \varepsilon) \\
\dot{y} & =B y+Y(\theta, x, y, \varepsilon)
\end{aligned}
$$

where $A$ and $B$ are constant $m \times m$ and $n \times n$ matrices, respectively, in Jordan canonical form;

$$
A=\operatorname{diag}\left(\lambda_{1}, \ldots, \lambda_{m}\right)+\operatorname{subdiag}\left(\gamma_{1}, \ldots, \gamma_{m-1}\right)
$$

with $\mathscr{R}\left(\lambda_{j}\right)<0$ and $\gamma_{j}=0$ or $\gamma(j=1, \ldots, m)$;

$$
B=\operatorname{diag}\left(\mu_{1}, \ldots, \mu_{n}\right)+\operatorname{subdiag}\left(\delta_{1}, \ldots, \delta_{n-1}\right)
$$

with $\mathscr{R}\left(\mu_{j}\right)>0$ and $\delta_{j}=0$ or $\delta(j=1, \ldots, n) ; \gamma$ and $\delta$ are sufficiently small positive constants; $\theta$ is a real scalar; $x$ and $y$ are complex vectors; $\varepsilon$ is a complex perturbation parameter ( $\varepsilon$ could be a vector; theorems and proofs would be similar); $\Theta, X, Y \in \Gamma^{1}(x, y, \varepsilon) ; X(\theta, x, y, 0), Y(\theta, x, y, 0) \in \Gamma^{2}(x, y)$.

THEOREM 4. There exist unique functions $x=\xi(\theta, \varepsilon)$ and $y=\eta(\theta, \varepsilon)$ with $\xi, \xi \theta, \eta$, $\eta_{\theta} \in \Gamma^{1}(\varepsilon)$ for which the $x$ and $y$ equations in (30) are satisfied. If system (30) is periodic (almost periodic) in $\theta$, then $\xi$ and $\eta$ also are periodic (almost periodic) in $\theta$ with the same period (frequencies).

REMARK. To be more precise one should say that

$$
M=\{(\theta, x, y) \mid \theta \text { arbitrary, } x=\xi(\theta, \varepsilon), y=\eta(\theta, \varepsilon)\}
$$

is an invariant manifold for system (30). On $M$ system (30) reduces to

$$
\dot{\theta}=1+\Theta(\theta, \xi(\theta, \varepsilon), \eta(\theta, \varepsilon)),
$$

and if $\psi(t)$ is any solution to this reduced equation, then $\theta=\psi(t), x=\xi(\psi(t), \varepsilon)$, $y=\eta(\psi(t), \varepsilon)$ is a solution of (30) lying on $M$. Since $\xi, \eta \in \Gamma^{1}(\varepsilon)$, it follows that 
$\xi(\psi(t), \varepsilon)$ and $\eta(\psi(t), \varepsilon)$ are bounded functions of $t$, and this in fact gives a characterization of $M$. The manifold $M$ is composed of those solutions of (30) which exist for all $t:-\infty<t<+\infty$, have their $(x, y)$ component bounded for all $t$, and remain near $\{(\theta, x, y) \mid \theta$ arbitrary, $(x, y)=0\}$ for all $t$.

If we eliminate $t$ in (30), we obtain

$$
\begin{aligned}
& \frac{d x}{d \theta}=A x+\cdots, \\
& \frac{d y}{d \theta}=B y+\cdots
\end{aligned}
$$

and for this system $x=\xi(\theta, \varepsilon), y=\eta(\theta, \varepsilon)$ represents the unique bounded solution having the property that $(\xi, \eta) \rightarrow 0$ uniformly in $\theta$ as $\varepsilon \rightarrow 0$. We have not used this approach because when $\theta$ has more than one dimension, it is not possible to eliminate $t$ in this manner. (This case will be treated in [4].)

Proof. Consider $\xi$ and $\eta$ as formal power series in $\varepsilon$ with undetermined coefficients. Since $\xi$ and $\eta$ satisfy the $x$ and $y$ equations of (30), we have

$$
\begin{aligned}
& \xi_{\theta}\{1+\Theta(\theta, \xi, \eta, \varepsilon)\}=A \xi+X(\theta, \xi, \eta, \varepsilon) \\
& \eta_{\theta}\{1+\Theta(\theta, \xi, \eta, \varepsilon)\}=B \eta+Y(\theta, \xi, \eta, \varepsilon)
\end{aligned}
$$

or equivalently,

$$
\begin{array}{ll}
\frac{\partial}{\partial \theta} \xi_{j}-\lambda_{j} \xi_{j}=\gamma_{j-1} \xi_{j-1}+X_{j}(\theta, \xi, \eta, \varepsilon)-\Theta(\theta, \xi, \eta, \varepsilon) \frac{\partial}{\partial \theta} \xi_{j} & (j=1, \ldots, m) \\
\frac{\partial}{\partial \theta} \eta_{j}-\mu_{j} \eta_{j}=\delta_{j-1} \eta_{j-1}+Y(\theta, \xi, \eta, \varepsilon)-\Theta(\theta, \xi, \eta, \varepsilon) \frac{\partial}{\partial \theta} \eta_{j} \quad(j=1, \ldots, n) .
\end{array}
$$

From (31) by means of Lemma 1 the coefficients of $\xi$ and $\eta$ can be computed recursively. To show convergence let

$$
\{1+\Theta(\theta, x, y, \varepsilon)\}^{-1}=1+\Theta *(\theta, x, y, \varepsilon) .
$$

Equations (31) can be written

$$
\begin{aligned}
\frac{\partial}{\partial \theta} \xi_{j}-\lambda_{j} \xi_{j}= & \left\{\gamma_{j-1} \xi_{j-1}+X_{j}(\theta, \xi, \eta, \varepsilon)\right\}\left\{1+\Theta^{*}(\theta, \xi, \eta, \varepsilon)\right\} \\
& +\lambda_{j} \xi_{j} \Theta^{*}(\theta, \xi, \eta, \varepsilon) \quad(j=1, \ldots, m) \\
\frac{\partial}{\partial \theta} \eta_{j}-\mu_{j} \eta_{j}= & \left\{\delta_{j-1} \eta_{j-1}+Y_{j}(\theta, \xi, \eta, \varepsilon)\right\}\left\{1+\Theta^{*}(\theta, \xi, \eta, \varepsilon)\right\} \\
& +\mu_{j} \eta_{j} \Theta *(\theta, \xi, \eta, \varepsilon) \quad(j=1, \ldots, n) .
\end{aligned}
$$

Choose $\Theta^{\prime}, X^{\prime}$, and $Y^{\prime}$ such that $\Theta^{*}(\theta, x, y, \varepsilon) \ll \Theta^{\prime}(x, y, \varepsilon), X(\theta, x, y, \varepsilon) \ll X^{\prime}(x, y, \varepsilon)$, and $Y(\theta, x, y, \varepsilon) \ll Y^{\prime}(x, y, \varepsilon)$; and such that the components of $X^{\prime}$ and $Y^{\prime}$ are all 
equal. Define $p$ and $q, \operatorname{dim} p=\operatorname{dim} \xi, \operatorname{dim} q=\operatorname{dim} \eta$, as formal power series in $\varepsilon$ with constant vector-valued coefficients by the following functional equations:

$$
\begin{array}{ll}
p_{j}=K\left[\left\{\gamma p_{j}+X_{j}^{\prime}(p, q, \varepsilon)\right\} \cdot\left\{1+\Theta^{\prime}(p, q, \varepsilon)\right\}+N p_{j} \Theta^{\prime}(p, q, \varepsilon)\right] & (j=1, \ldots, m) \\
q_{j}=K\left[\left\{\delta q_{j}+Y_{j}^{\prime}(p, q, \varepsilon)\right\} \cdot\left\{1+\Theta^{\prime}(p, q, \varepsilon)\right\}+N q_{j} \Theta^{\prime}(p, q, \varepsilon)\right] & (j=1, \ldots, n) .
\end{array}
$$

$K$ and $N$ are large positive constants. If $K \gamma$ and $K \delta$ are less than 1, then the implicit function theorem proves the existence of $p=p(\varepsilon), q=q(\varepsilon)$, analytic in $\varepsilon$ in some neighborhood of the origin and vanishing at the origin. Using Lemma 1 it is easy to show by induction that $\xi(\theta, \varepsilon) \ll p(\varepsilon)$ and $\eta(\theta, \varepsilon) \ll q(\varepsilon)$, provided $K$ and $N$ are sufficiently large, their size being determined only by the eigenvalues of $A$ and $B$. By choosing $\gamma$ and $\delta$ sufficiently small, $K \gamma$ and $K \delta$ will be less than 1 . Thus $\xi, \eta \in \Gamma^{1}(\varepsilon)$ and by (32), $\xi_{\theta}, \eta_{\theta} \in \Gamma^{1}(\varepsilon)$. The remarks on periodicity and almost periodicity follow from Lemma 1 . This completes the proof of Theorem 4.

Let $z=(x, y)$ and $\zeta=(\xi, \eta)$. By introducing the new variable

$$
p=z-\zeta(\theta, \varepsilon)
$$

we obtain from (30) and the equations above (31) an equation of the form

$$
d p / d \theta=(E+F(\theta, \varepsilon)) p+P(\theta, p, \varepsilon)
$$

where $E=\operatorname{diag}(A, B) ; F \in \Gamma^{1}(\varepsilon) ; P \in \Gamma^{2}(p, \varepsilon) \cap \Gamma^{2}(p)$. At this point we need Sibuya's linear change of variables.

Although $E=\operatorname{diag}(A, B)$ in $(34)$, it will be useful to consider a more general situation in Theorem 5 below. Consider the linear system

$$
d p / d \theta=(E+F(\theta, \varepsilon)) p
$$

where $E=\operatorname{diag}\left(E_{11}, \ldots, E_{n n}\right) ; E_{j j}(j=1, \ldots, n)$ are constant square matrices not necessarily of the same dimension; the maximum of the real parts of the eigenvalues of $E_{j j}$ is less than the minimum of the real parts of the eigenvalues of $E_{k k}$ when $j<k ; F \in \Gamma^{1}(\varepsilon)$.

THEOREM 5. For system (35) there exists a unique linear change of variables

$$
p=(I+G(\theta, \varepsilon)) q
$$

such that

$$
d q / d \theta=(E+H(\theta, \varepsilon)) q
$$

where $I$ is the identity matrix; $G_{j j} \equiv 0(j=1, \ldots, n) ; H=\operatorname{diag}\left(H_{11}, \ldots, H_{n n}\right)$; $G, G_{\theta}, H \in \Gamma^{1}(\varepsilon)$. If (35) is real, so are (36) and (37). If $F$ is periodic (almost periodic) in $\theta$, then $G$ and $H$ are periodic (almost periodic) in $\theta$ with the same period (frequencies).

Here we are using a partition of the matrices $G$ and $H$ which corresponds to the partition of $E$. For a proof of Theorem 5 see [7, pp. 128-145]. 
Now, if in (34) we introduce the linear change of variables

$$
p=(I+G(\theta, \varepsilon)) q
$$

as described in Theorem 5 with respect to the linear part of (34), we obtain an equation of the type

$$
d q / d \theta=(E+H(\theta, \varepsilon)) q+Q(\theta, q, \varepsilon)
$$

where $H=\operatorname{diag}\left(H_{11}, H_{22}\right) ; \operatorname{dim} H_{11}=\operatorname{dim} A$ and $\operatorname{dim} H_{22}=\operatorname{dim} B ; H \in \Gamma^{1}(\varepsilon)$; $Q \in \Gamma^{2}(q, \varepsilon) \cap \Gamma^{2}(q)$. Setting $q=(r, s)$ where $\operatorname{dim} r=\operatorname{dim} x$ and $\operatorname{dim} s=\operatorname{dim} y$, we obtain from (38) a system of the type

$$
\begin{aligned}
\dot{\theta} & =1+\Phi(\theta, r, s, \varepsilon) \\
\dot{r} & =\left(A+A^{0}(\theta, \varepsilon)\right) r+R(\theta, r, s, \varepsilon) \\
\dot{s} & =\left(B+B^{0}(\theta, \varepsilon)\right) s+S(\theta, r, s, \varepsilon)
\end{aligned}
$$

where $\Phi \in \Gamma^{1}(r, s, \varepsilon) ; A^{0}, B^{0} \in \Gamma^{1}(\varepsilon) ; R, S \in \Gamma^{2}(r, s, \varepsilon) \cap \Gamma^{2}(r, s)$.

THEOREM 6. For system (39) there exists a change of variables

$$
\begin{aligned}
& u=r-M(\theta, r, s, \varepsilon) \\
& v=s-N(\theta, r, s, \varepsilon)
\end{aligned}
$$

such that

$$
\begin{aligned}
\dot{u} & =\left(A+A^{0}(\theta, \varepsilon)\right) u+U(\theta, u, v, \varepsilon) \\
\dot{v} & =\left(B+B^{0}(\theta, \varepsilon)\right) v+V(\theta, u, v, \varepsilon) \\
U(\theta, 0, v, \varepsilon) & \equiv 0, \quad U(\theta, u, 0, \varepsilon)=\tilde{U}(\theta, u, \varepsilon) \\
V(\theta, 0, v, \varepsilon) & =\tilde{V}(\theta, v, \varepsilon), \quad V(\theta, u, 0, \varepsilon) \equiv 0
\end{aligned}
$$

where

$$
M, M_{\theta}, N, N_{\theta} \in \Gamma^{2}(r, s, \varepsilon) \cap \Gamma^{2}(r, s) ; U, V \in \Gamma^{2}(u, v, \varepsilon) \cap \Gamma^{2}(u, v) .
$$

Assuming that the eigenvalues of $A$ and $B$ are ordered so that

$$
\mathscr{R}\left(\lambda_{m}\right) \leqq \cdots \leqq \mathscr{R}\left(\lambda_{1}\right)<0<\mathscr{R}\left(\mu_{1}\right) \leqq \cdots \leqq \mathscr{R}\left(\mu_{n}\right),
$$

then the components of $\tilde{U}$ are polynomials .... (The remainder of the statement of the theorem parallels the statement of Theorem 3.)

Proof. Since (40) represents a composition of changes of variables similar to the situation occurring in Theorem 3, we will only show the first step. Namely, rather than (40) we will find a change of variables

$$
w=s-Q(\theta, r, \varepsilon)
$$

such that

$$
\dot{w}=\left(B+B^{0}(\theta, \varepsilon)\right) w+W(\theta, r, w, \varepsilon), \quad W(\theta, r, 0, \varepsilon) \equiv 0
$$


where $Q, Q_{\theta} \in \Gamma^{2}(r, \varepsilon) \cap \Gamma^{2}(r) ; W \in \Gamma^{2}(r, w, \varepsilon) \cap \Gamma^{2}(r, w)$. As in Theorem 3, all other changes of variables necessary to effect (41) are similar to this one. The equation that $Q$ must satisfy is

$$
Q_{\theta}\{1+\Phi(\theta, r, Q, \varepsilon)\}+Q_{r}\left\{\left(A+A^{0}\right) r+R(\theta, r, Q, \varepsilon)\right\}=\left(B+B^{0}\right) Q+S(\theta, r, Q, \varepsilon)
$$

or equivalently,

$$
\begin{aligned}
\frac{\partial}{\partial \theta} Q_{j}+\sum_{l=1}^{m} \lambda_{l} r_{l} \frac{\partial}{\partial r_{l}} Q_{j}-\mu_{j} Q_{j} \\
=\gamma_{j-1} Q_{j-1}-\sum_{l=1}^{m} \delta_{l-1} r_{l-1} \frac{\partial}{\partial r_{l}} Q_{j}-\sum_{k, l=1}^{m} a_{l k}^{0} r_{k} \frac{\partial}{\partial r_{l}} Q_{j} \\
+\sum_{l=1}^{n} b_{j l}^{0} Q_{l}-\Phi(\theta, r, Q, \varepsilon) \frac{\partial}{\partial \theta} Q_{j}+S_{j}(\theta, r, Q, \varepsilon) \\
\\
-\sum_{l=1}^{n} R_{l}(\theta, r, Q, \varepsilon) \frac{\partial}{\partial r_{l}} Q_{j} \quad(j=1, \ldots, n)
\end{aligned}
$$

where $A^{0}=\left(a_{l k}^{0}\right)$ and $B^{0}=\left(b_{j l}^{0}\right)$. Following along an order relation similar to $\prec$ (one must take into account that $Q$ is now a power series in $(r, \varepsilon)$ rather than just $r$ ), the coefficients of $Q$ can be computed recursively from (42) with the aid of Lemma 1. Choose $A^{\prime}, B^{\prime}, \Phi^{\prime}, R^{\prime}$, and $S^{\prime}$ such that

$$
\begin{gathered}
A^{0}(\theta, \varepsilon) \ll A^{\prime}(\varepsilon), B^{0}(\theta, \varepsilon) \ll B^{\prime}(\varepsilon), \Phi(\theta, r, s, \varepsilon) \ll \Phi^{\prime}(r, s, \varepsilon), \\
R(\theta, r, s, \varepsilon) \ll R^{\prime}(r, s, \varepsilon) \text { and } S(\theta, r, s, \varepsilon) \ll S^{\prime}(r, s, \varepsilon) .
\end{gathered}
$$

In addition we require that the components of $A^{\prime}$ are all equal, the components of $B^{\prime}$ are all equal, the components of $R^{\prime}$ are all equal, and that the components of $S^{\prime}$ are all equal. Let $Q^{\prime}=Q^{\prime}(r, \varepsilon), \operatorname{dim} Q^{\prime}=\operatorname{dim} Q$, be the formal power series in $(r, \varepsilon)$ defined by the functional equation

$$
\begin{gathered}
\sum_{l=1}^{m} r_{l} \frac{\partial}{\partial r_{l}} Q_{j}^{\prime}=K\left[\gamma_{j-1} Q_{j-1}^{\prime}+\sum_{l=1}^{m} \delta_{l-1} r_{l-1} \frac{\partial}{\partial r_{l}} Q_{j}^{\prime}+\sum_{k, l=1}^{m} a_{l k}^{\prime} r_{k} \frac{\partial}{\partial r_{l}} Q_{j}\right. \\
+\sum_{l=1}^{n} b_{j l}^{\prime} Q_{l}^{\prime}+\Phi^{\prime}\left(r, Q^{\prime}, \varepsilon\right) \sum_{l=1}^{m} r_{l} \frac{\partial}{\partial r_{l}} Q_{j}^{\prime}+S_{j}^{\prime}\left(r, Q^{\prime}, \varepsilon\right) \\
\left.+\sum_{l=1}^{m} R_{l}^{\prime}\left(r, Q^{\prime}, \varepsilon\right) \frac{\partial}{\partial r_{l}} Q_{j}^{\prime}\right] \quad(j=1, \ldots, n) .
\end{gathered}
$$

As in Theorem 1, with Lemmas 1 and 2 one establishes that

$$
Q(\theta, r, \varepsilon) \ll Q^{\prime}(r, \varepsilon) .
$$

Let $\xi$ and $\eta$ be one dimensional variables. When we put $\xi=\eta=r_{1}+\cdots+r_{m}+\varepsilon$ it is clear that

$$
Q^{\prime}\left(r_{1}, \ldots, r_{m}, \varepsilon\right) \ll Q^{\prime}(\xi, \ldots, \xi, \eta)
$$


Let $\Phi^{\prime \prime}(\xi, s, \eta)=\Phi^{\prime}(\xi, \ldots, \xi, s, \eta)$ and similarly define $R^{\prime \prime}$ and $S^{\prime \prime}$ when we have replaced $r_{l}(l=1, \ldots, m)$ by $\xi$ and $\varepsilon$ by $\eta$ in $\Phi^{\prime}, R^{\prime}$, and $S^{\prime}$. Define $A^{\prime \prime}=A^{\prime \prime}(\eta)=A^{\prime}(\eta)$ and $B^{\prime \prime}=B^{\prime \prime}(\eta)=B^{\prime}(\eta)$ when we have replaced $\varepsilon$ by $\eta$ in $A^{\prime}$ and $B^{\prime}$. Define $Q^{\prime \prime}=Q^{\prime \prime}(\xi, \eta), \operatorname{dim} Q^{\prime \prime}=\operatorname{dim} Q^{\prime}$, by the functional equation

$$
\begin{aligned}
\xi Q_{\xi}^{\prime \prime}-Q^{\prime \prime}=2 K\left[A^{\prime \prime} \xi Q_{\xi}^{\prime \prime}+B^{\prime \prime} Q^{\prime \prime}+\Phi^{\prime \prime}(\xi,\right. & \left.Q^{\prime \prime}, \eta\right) \xi Q_{\xi}^{\prime \prime} \\
& \left.+S^{\prime \prime}\left(\xi, Q^{\prime \prime}, \eta\right)+R_{1}^{\prime \prime}\left(\xi, Q^{\prime \prime}, \eta\right) Q_{\xi}^{\prime \prime}\right] .
\end{aligned}
$$

Since the components of $A^{\prime \prime}$ are all equal, and similarly for $B^{\prime \prime}, R^{\prime \prime}$, and $S^{\prime \prime}$; one observes that the components of $Q^{\prime \prime}$ are all equal. Hence, using the techniques of Theorem 1, it follows that

$$
Q^{\prime}(\xi, \ldots, \xi, \eta) \ll Q^{\prime \prime}(\xi, \eta) .
$$

Up to this point we have paralleled the arguments of Theorem 1, but to complete our proof we must introduce a technique used by C. L. Siegel in [9]. From (44) we write

$$
\xi Q_{\xi}^{\prime \prime}-Q^{\prime \prime}=F Q_{\xi}^{\prime \prime}+G
$$

where $F Q_{\xi}^{n}$ represents all terms with $Q_{\xi}^{n}$ as a factor and $G$ represents the remaining terms. Let

$$
Q^{\prime \prime}=\sum_{\alpha=2}^{\infty} \sum_{\beta=0}^{\infty} Q_{\alpha, \beta}^{\prime \prime} \xi^{\alpha} \eta^{\beta}
$$

From (45) we have

$$
\sum_{\alpha=2}^{\infty} \sum_{\beta=0}^{\infty}(\alpha-1) Q_{\alpha, \beta}^{\prime \prime} \xi^{\alpha} \eta^{\beta}=F \sum_{\alpha=2}^{\infty} \sum_{\beta=0}^{\infty} \alpha Q_{\alpha, \beta}^{\prime \prime} \xi^{\alpha-1} \eta^{\beta}+G
$$

If we equate the $(\mu, v)$ th coefficient on both sides of $(46)$, we obtain

$$
\begin{aligned}
& =\left[F \sum_{\alpha=2}^{\mu} \sum_{\beta=0}^{\nu} \frac{\alpha}{\mu-1} Q_{\alpha, \beta}^{\prime \prime} \xi^{\alpha-1} \eta^{\beta}+\frac{1}{\mu-1} G\right]_{\mu, v} \\
& \leqq 2\left[F \sum_{\alpha=2}^{\mu} \sum_{\beta=0}^{\nu} Q_{\alpha, \beta}^{\prime \prime} \xi^{\alpha-1} \eta^{\beta}+G\right]_{\mu, \nu}
\end{aligned}
$$

where $[\cdots]_{\mu, v}$ represents the coefficient of $\xi^{\mu} \eta^{\nu}$ of the total expansion (one must expand $F$ and $G$, multiply and collect terms) in the brackets. Therefore it follows that

$$
Q^{\prime \prime}(\xi, \eta) \ll \tilde{Q}(\xi, \eta)
$$

where $\widetilde{Q}$ is defined by the functional equation

$$
\tilde{Q}=4 K\left[\left(A^{\prime \prime}+B^{\prime \prime}\right) \tilde{Q}+\Phi^{\prime \prime}(\xi, \tilde{Q}, \eta) \tilde{Q}+S^{\prime \prime}(\xi, \tilde{Q}, \eta)+R_{1}^{\prime \prime}(\xi, \tilde{Q}, \eta) \xi^{-1} \tilde{Q}\right]
$$


Let $\tilde{Q}=\xi Q^{*}$, then

$$
\begin{gathered}
Q^{*}=4 K\left[\left(A^{\prime \prime}+B^{\prime \prime}\right) Q^{*}+\Phi^{\prime \prime}\left(\xi, \xi Q^{*}, \eta\right) Q^{*}+\xi^{-1} S^{\prime \prime}\left(\xi, \xi Q^{*}, \eta\right)\right. \\
\left.+\xi^{-1} R_{1}^{\prime \prime}\left(\xi, \xi Q^{*}, \eta\right) Q^{*}\right] .
\end{gathered}
$$

Since $S^{\prime \prime}(\xi, s, \eta), R_{1}^{\prime \prime}(\xi, s, \eta) \in \Gamma^{2}(\xi, s, \eta) \cap \Gamma^{2}(\xi, s)$, the convergence of $Q^{*}$ in a neighborhood of $\xi=\eta=0$ is an immediate consequence of the implicit function theorem. This completes the proof of Theorem 6 .

In order to develop a perturbation theory for systems of the type considered in Theorem 3, we require periodicity in $\theta$ and a restriction on the purely imaginary eigenvalues. Consider the system

$$
\begin{aligned}
\dot{\theta} & =1+\Theta(\theta, x, y, z, \varepsilon) \\
\dot{x} & =A x+X(\theta, x, y, z, \varepsilon) \\
\dot{y} & =B y+Y(\theta, x, y, z, \varepsilon) \\
\dot{z} & =C z+Z(\theta, x, y, z, \varepsilon)
\end{aligned}
$$

where $\Theta, X, Y$, and $Z$ have period $\omega$ in $\theta ; A, B$, and $C$ are the matrices in (28) with the additional property that no eigenvalue of $B$ is equal to $2 \pi i \omega^{-1} \times$ integer; $\theta$ is a real scalar; $x, y$, and $z$ are complex vectors; $\varepsilon$ is a perturbation parameter; $\Theta, X, Y, Z \in \Gamma^{1}(x, y, z, \varepsilon) ; X(\theta, x, y, z, 0), Y(\theta, x, y, z, 0), Z(\theta, x, y, z, 0) \in \Gamma^{2}(x, y, z)$.

THEOREM 7. For system (47) there exists a change of variables

$$
\begin{aligned}
u & =x-\xi(\theta, \varepsilon)-P(\theta, x, y, z, \varepsilon) \\
v & =y-\eta(\theta, \varepsilon)-Q(\theta, x, y, z, \varepsilon) \\
w & =z-\zeta(\theta, \varepsilon)-R(\theta, x, y, z, \varepsilon)
\end{aligned}
$$

such that

$$
\begin{aligned}
\dot{u} & =\left(A+A^{0}(\theta, \varepsilon)\right) u+U(\theta, u, v, w, \varepsilon) \\
\dot{v} & =\left(B+B^{0}(\theta, \varepsilon)\right) v+V(\theta, u, v, w, \varepsilon) \\
\dot{w} & =\left(C+C^{0}(\theta, \varepsilon)\right) w+W(\theta, u, v, w, \varepsilon) \\
U(\theta, 0,0, w, \varepsilon) & \equiv 0, \quad U(\theta, u, 0,0, \varepsilon)=\tilde{U}(\theta, u, \varepsilon) \\
V(\theta, 0,0, w, \varepsilon) & \equiv V(\theta, u, 0,0, \varepsilon) \equiv 0 \\
W(\theta, 0,0, w, \varepsilon) & =\tilde{W}(\theta, w, \varepsilon), \quad W(\theta, u, 0,0, \varepsilon) \equiv 0
\end{aligned}
$$

where $\xi, \xi_{\theta}, \eta, \eta_{\theta}, \zeta, \zeta_{\theta} \in \Gamma^{1}(\varepsilon) ; P, P_{\theta}, Q, Q_{\theta}, R, R_{\theta} \in \Gamma^{2}(x, y, z, \varepsilon) ; A^{0}, B^{0}, C^{0} \in \Gamma^{1}(\varepsilon)$; $U, V, W \in \Gamma^{2}(u, v, w, \varepsilon) \cap \Gamma^{2}(u, v, w)$; the components of $\tilde{U}$ are polynomials.... (The remainder of the statement of the theorem parallels the statement of Theorem 3 restricted to the periodic (or constant) case.) 
Proof. The change of variables (48) represents a composition of changes of variables, the situation being somewhat more involved than in Theorem 3. However, only one technique is needed here which has not been discussed above. The functions $\xi, \eta$, and $\zeta$ in (48) satisfy the $x, y$, and $z$ equations in (47). If we assume that $\xi, \eta$, and $\zeta$ are formal power series with undetermined coefficients, then from the equations

$$
\begin{aligned}
\xi_{\theta}-A \xi & =X-\Theta \xi_{\theta} \\
\eta_{\theta}-B \eta & =Y-\Theta \eta_{\theta} \\
\zeta_{\theta}-C \zeta & =Z-\Theta \zeta_{\theta}
\end{aligned}
$$

one can compute the coefficients of $\xi, \eta$, and $\zeta$ recursively. Since $A$ has eigenvalues with negative real parts and $C$ has eigenvalues with positive real parts, the coefficients of $\xi$ and $\zeta$ can be computed as in Theorem 4. Let

$$
\eta(\theta, \varepsilon)=\sum_{\alpha=1}^{\infty} \eta_{\alpha}(\theta) \varepsilon^{\alpha}
$$

By equating coefficients of $\varepsilon$, one obtains equations of the form

$$
\partial \eta_{v} / \partial \theta-B \eta_{v}=f_{v} \quad(\nu=1,2, \ldots)
$$

where $f_{v}$ is a known function of $\theta$ with period $\omega$ when all the coefficients $\xi_{\alpha}, \eta_{\alpha}$, $\zeta_{\alpha}, \alpha<\nu$, have been computed. The unique solution of (49) having period $\omega$ in $\theta$ is given by

$$
\eta_{\nu}(\theta)=\left(e^{-B \omega}-I\right)^{-1} \int_{0}^{\omega} e^{-B \sigma} f_{\nu}(\theta+\sigma) d \sigma
$$

The hypothesis that no eigenvalue of $B$ is equal to $2 \pi i \omega^{-1} \times$ integer assures us that $\left(e^{-B \omega}-I\right)$ is nonsingular.

The convergence of the formal power series $\xi, \eta, \zeta$ is accomplished along the lines of Theorem 4. Using Theorem 5 and the techniques developed in the proof of Theorem 6, one can now complete the proof of Theorem 7 .

\section{BIBLIOGRAPHY}

1. Ė. Belaga, The reducibility of a system of ordinary differential equations in the neighborhood of a conditionally periodic motion, Dokl. Akad. Nauk SSSR 143 (1962), 255-258 = Soviet Math. Dokl. 3 (1962), 360-364.

2. H. Dulac, Solutions d'un système d'equations différentielles dans le voisinage de valeurs singulières, Bull. Soc. Math. France 40 (1912), 324-383.

3. A. Kelley, Change of variables techniques in ordinary differential equations, Thesis, Univ. of California, Berkeley, 1963.

4. - Changes of variables near a periodic surface or integral manifold, (to appear).

5. S. Lefschetz, Differential equations: geometric theory, Interscience, New York, 1957.

6. H. Poincaré, Oeuvres de Henri Poincaré, Tome I, Gauthier-Villars, Paris, 1951. 
7. Y. Sibuya, Non-linear ordinary differential equations with periodic coefficients, Funkcial. Ekvac. 1 (1958), 121-205.

8. - On bounded solutions of ordinary differential equations with almost periodic coefficients, Symposium Internacional de Ecuaciones Diferenciales Ordinarias, University of Mexico, 1961, pp. 290-293.

9. C. L. Siegel, Der Dreierstoss, Ann. of Math. 42 (1941), 127-168.

10. - Uber die Normalform analytischer Differentialgleichungen in der Nähe einer Gleichgewichtslösung, Nachr. Akad. Wiss. Göttingen Math.-Phys. Kl. IIa 5 (1952), 21-30.

\author{
INSTITUTE FOR ADVANCED STUdY, \\ Princeton, New Jersey \\ UNIVERSITY OF CALIFORNIA, \\ Santa Cruz, California
}

